\title{
T-bet acts as a powerful adjuvant in Ag85B DNA-based vaccination against tuberculosis
}

\author{
DONG HU, JING WU, RONGBO ZHANG and LIPING CHEN \\ Department of Medical Immunology, Medical School and Institute of Infection and Immunity, \\ Anhui University of Science and Technology, Huainan, Anhui 232001, P.R. China
}

Received January 13, 2012; Accepted April 3, 2012

DOI: $10.3892 / \mathrm{mmr} .2012 .883$

\begin{abstract}
Owing to the limitations of traditional Bacillus Calmette-Guerin vaccines and the low efficacy of DNA vaccines expressing single antigens of Mycobacterium tuberculosis, there is a pressing requirement for adjuvants capable of strengthing the immunogenicity and effectiveness of these vaccines against tuberculosis (TB). T-bet (TBX21) is a transcription factor, which controls the optimal development of type-1 immune responses responsible for the potent protection of vaccines against TB. However, little is known about the efficiency of the TB vaccine combined with T-bet. In this study, we report an approach to intensify the immunogenicity of Ag85B DNA-based vaccines using T-bet as an adjuvant. Balb/c mice were immunized by 3 intramuscular inoculations with pcDNA3.1-FLAG-T-bet in combination with pcDNA3.1FLAG-Ag85B, and the immune responses were compared with those induced by vaccination with Ag85B DNA alone. We found that pcDNA3.1-T-bet-Ag85B not only induced evidently higher IgG2a antibody responses, but also increased the production of interferon- $\gamma$ (IFN- $\gamma$ ) and interleukin (IL)-2 with the concomitant repression of IL-4 and IL-10 compared with pcDNA3.1-Ag85B alone or the empty vector. Thus, plasmid DNA coding for T-bet enhanced Ag85B-specific immune responses and shifted them to a predominant Th1-type immune response. In conclusion, T-bet is an efficacious Th1-inducing adjuvant in the context of the Ag85B DNA-based vaccination, and could also prove to be a promising candidate for DNA vaccine development against TB.
\end{abstract}

Correspondence to: Professor Rongbo Zhang or Dr Jing Wu, Department of Medical Immunology, Medical School and Institute of Infection and Immunity, Anhui University of Science and Technology, No. 25 Middle Dongshan Road, Huainan, Anhui 232001, P.R. China

E-mail: lory456@126.com

E-mail:wujing8008@126.com

Key words: T-bet, Ag85B, vaccine, Mycobacterium tuberculosis, cytokines, adjuvants

\section{Introduction}

Tuberculosis (TB) remains a major infectious disease worldwide. Infection with Mycobacterium tuberculosis (MTB) can cause $\mathrm{T}$ helper (Th)-1-type immune responses and delayed-type hypersensitivity mediated by $\mathrm{T}$ cells and macrophages. Infections that occur during the late period result in a reduced Th1 response, accompanied by an increased Th2 response $(1,2)$. Furthermore, antigen-specific Th1 responses are vital for the prevention and inhibition of MTB infection. Currently, Bacillus Calmette-Guerin (BCG), a live attenuated Mycobacterium bovis, which is is commonly used in a number of countries as the only available TB vaccine, has been shown to induce strong Th1-type immune responses (3). However, over the years, the limitations of the BCG vaccine have become evident, including the inconsistent effects of the vaccination on the immune system, as well as the safety and diagnosis limitations (4). Therefore, there is a pressing requirement for developing safe, cost-effective vaccines, such as DNA vaccines, as an alternative to the BCG vaccine in order to achieve powerful immune responses against TB.

Understanding of the immunogenicity of and the protective mechanism against MTB is crucial for the development of new and effective vaccines. In previous years, the secreted MTB protein, Ag85B, has been the main focus of intensive research. $\mathrm{Ag} 85 \mathrm{~B}$, a $30-\mathrm{kDa}$ fibronectin-binding protein with mycolyl-transferase activity, is a major protein secreted by all Mycobacterium species belonging to the Ag85B family and is a potent immunoprotective antigen (5). It has also been demonstrated to induce a powerful Th1-type immune response in mice as well as in humans, and to induce Th1-type cells to produce interleukin (IL)-2, tumor necrosis factor- $\alpha$ (TNF- $\alpha$ ) and interferon- $\gamma($ IFN- $\gamma$ ). A number of studies have demonstrated a significant protective effect in the lungs of mice immunized with $\mathrm{Ag} 85 \mathrm{~B}(6,7)$, while there are only a few studies contradicting the efficacy of the Ag85B vaccine $(5,8,9)$. Thus, in order to further identify the immunoprotective force of DNA vaccines against TB, the appropriate immune adjuvant is required.

Although adjuvants, such as Freund's adjuvant, are able to elicit Th1-type immune responses to a certain extent, they cannot be used in humans as they are highly toxic; thus to date there is no adjuvant which is able to induce a strong cellular response. No single adjuvant is sufficient for broad application 
and therefore tailor-made adjuvants for specific vaccines are required.

T-bet is a member of the T-box family of transcription factors encoding 530 amino acids which appears to regulate lineage commitment in CD4 Th lymphocytes by activating the hallmark Th1 cytokine, IFN- $\gamma$. Therefore, T-bet is also known as a Th1-specific transcription factor. IFN- $\gamma$ is also produced by natural killer (NK) cells and most prominently by CD 8 cytotoxic T cells, and is vital for the control of microbial pathogens. A previous study indicated that dendritic cells (DCs), as well as T cells and NK cells, are able to secrete large amounts of IFN- $\gamma$ (10). T-bet is the key factor in controlling IFN- $\gamma$ secretion by DCs. There is no apparent impairment in the development, differentiation and activation of DCs in T-bet ${ }^{-1-}$ mice. However, T-bet ${ }^{--}$DCs only secrete trace quantities of IFN- $\gamma$ in the context of IL-2 alone or in combination with IL-8, which negatively affects their ability to activate Th1 and induce antigen-specific cytotoxic T lymphocytes (CTLs) $(11,12)$. However, this effect can be reversed by using T-bet transfected into DCs. Additionally, the in vitro overexpression of T-bet in B cell lines or the originally generated cells can lead to antibody class switching, which causes the level of $\operatorname{IgG} 2 \mathrm{a}$ to increase significantly. By contrast, the deletion of T-bet in mice causes the loss of IgG2a, IgG2b and IgG3 antibodies, and leads to a predominant Th2-type immune response. These results demonstrate that T-bet plays a crucial role in Th1-type immune differentiation, and converts the Th2-biased immune response to a Th1-type with a large amount of IFN- $\gamma$ production (13).

Therefore, in this study, we aimed to construct a T-bet plasmid as a genetic adjuvant for an Ag85B DNA-based vaccine, namely a combination/composite vaccine, as well as to determine the immunogenicity of the T-bet-Ag85B vaccine and immune responses compared with $\mathrm{Ag} 85 \mathrm{~B}$ alone.

\section{Materials and methods}

Plasmids. A virulent MTB H37Rv strain (ATCC 35718) was kindly provided by Dr W. Chen (The 8th People's Hospital of Guangzhou, Guangzhou, China). The pcDNA3.1-FLAG plasmid was a gift from Dr Wang Fang (Zhongshan School of Medicine, Sun Yat-Sen University, Guangzhou, China). As described previously $(14,15)$, the target gene fragments encoding the mature Ag85B were obtained by RT-PCR amplification using genomic DNA from the MTB H37Rv strain as the template with the specific primers, P1 (GCAAGCTTATGAC AGACGTGAGCCGA) and P2 (GCGGATCCCTAGGCAGC ATCGAGTC). The target gene fragments coding for T-bet were amplified by RT-PCR using total RNA extracted from the splenic lymphocytes of mice as the template with the specific primers, P1 (GTCTCGAGCACCATGGGCATCGT GGAGCCGGGTT) and P2 (GCGAATTCACTGTTTCTG TTCCTTTCATCATGTCATCTGCT). Restriction enzyme sites are marked as italicized and underlined. The PCR products encoding the Ag85B and T-bet were digested with Hind III, BamHI and XhoI, EcoRI restriction enzymes, respectively. They were then subcloned into the pMD18-T vector digested with the endonucleases, and inserted into the corresponding sites of the eukaryotic expression vector, pcDNA3.1-FLAG. These recombinants were identified by DNA sequencing. Recombinant plasmid DNAs for vaccination were prepared and purified using the Plasmid Maxi_kit, according to the manufacturer's instructions, and suspended in endotoxin-free physiologic saline. They were then stored at $-20^{\circ} \mathrm{C}$ until use.

Transient transfection and reporter gene assay. Raw 264.7 cells were cultured in Dulbecco's $\mu$ odified Eagle's medium (DMEM) containing 10\% fetal calf serum (FCS) and antibiotics. When they reached a confluence level of approximately $70-80 \%$, the cells were plated overnight in 6-well plates containing glass cover-slips. Transient transfections were performed using Lipofectamine 2000 reagent, as previously described (16). For transactivation assays 1, 2 and $4 \mu \mathrm{g} / \mathrm{ml}$ pcDNA3.1-FLAG-T-bet and pcDNA3.1-FLAG-Ag85B expression vectors were transfected into Raw 264.7 cells, using $\beta$-actin as the internal control. Two days after transfection, the cells were harvested, washed with PBS and then lysed with lysis buffer on ice for $30 \mathrm{~min}$ with vortexing. After centrifugation at $15,000 \mathrm{x}$ g for $15 \mathrm{~min}$, the supernatants were collected and subjected to western blot analysis. Aliquots of whole cell extracts were separated on a $10 \%$ SDS-PAGE gel and transferred to a polyvinylidene difluoride membrane. Western blot analysis was performed by probing the blots with polyclonal antibodies against FLAG and actin.

Detection of anti-Ag85B antibodies. Female Balb/c mice (6-8 weeks old) were purchased from the animal center of Anhui University of Science and Technology and raised carefully in accordance with the National Institutes of Health guidelines on animal care. All experimental procedures were approved by the Animal Care And Use Committee of Anhui University of Science and Technology (Permit numbers: AUST 2011-0011). Balb/c mice were randomly divided into 4 groups: the pcDNA3.1-T-bet in combination with pcDNA3.1-Ag85B group (T-bet + Ag85B), the pcDNA3.1-T-bet group (T-bet), the pcDNA3.1-Ag85B group (Ag85B) and the pcDNA3.1 empty vector group (control). The mice were then vaccinated by 3 intramuscular inoculations. Two weeks later, the titers of antiAg85B IgG1 and IgG2a antibodies in the sera from immunized mice were detected by ELISA using recombinant Ag85B purified by our laboratory as previously described (17). In brief, $5 \mu \mathrm{g} / \mathrm{ml}$ recombinant $\mathrm{Ag} 85 \mathrm{~B}$ protein in carbonate-bicarbonate buffer were incubated in 96-well plates overnight at $4^{\circ} \mathrm{C}$. Next, $5 \%$ bovine serum albumin in PBS was added for $2 \mathrm{~h}$ at $37^{\circ} \mathrm{C}$. Then, individual serum samples were added in duplicate and incubated for $1 \mathrm{~h}$ at $37^{\circ} \mathrm{C}$. Subsequently, the bound antibodies were detected with 1:500 diluted HRP-conjugated goat antimouse IgG1 and IgG2a (Sigma, St. Louis, MO, USA) for $1 \mathrm{~h}$ at $37^{\circ} \mathrm{C}$, respectively. After washing, TMB substrate was added to the individual wells, and the reaction was stopped by adding $1 \mathrm{M} \mathrm{H}_{2} \mathrm{SO}_{4}$. The optical density (OD) was measured at $450 \mathrm{~nm}$.

Cytokine release assay. Two weeks after the last immunization, the mice were sacrificed and their splenic lymphocytes were prepared by a routine method. Splenocytes from individual mice were stimulated in duplicate with $10 \mu \mathrm{g} / \mathrm{ml} \mathrm{Ag85B}$ in 24-well plates. After $72 \mathrm{~h}$, culture supernatants were harvested and the presence of cytokines, including IFN- $\gamma$, IL-2, IL-4 and IL-10, were detected using commercial mouse cytokine immunoassay ELISA kits (R\&D Systems, Minneapolis, MN, 
USA), according to the manufacturer's instructions, as previously described (18).

MTB infection and colony-forming unit (CFU) determination. Three months after the last immunization, different groups of mice were challenged intravenously at the tail vein with $1 \times 10^{5} \mathrm{CFU}$ of the MTB H37Rv strain. The mice spleens and lungs were dissected on weeks $1,2,4$ and 8 . One portion of the spleen and lung tissues was homogenized and subjected to serial dilutions. An aliquot was plated on solid Middlebrook $7 \mathrm{H} 10$ agar plates and incubated for 4 weeks at $37^{\circ} \mathrm{C}$. The colonies were counted and the results were expressed as $\log _{10} \mathrm{CFU}$.

Statistical analysis. Data are presented as the means \pm SD. The unpaired two-tailed Student's t-test was used to determine significant differences between the groups. $\mathrm{P}<0.05$ or $\mathrm{P}<0.01$ was considered to indicate a statistically significant difference.

\section{Results}

Expression of DNA vaccine plasmids in vitro. To determine whether pcDNA3.1-FLAG-T-bet or pcDNA3.1-FLAG-Ag85B were expressed in vitro, Raw 264.7 cells were transiently transfected with the plasmid constructs. Protein expression was detected by western blot analysis with the evidently expressed bonds probed by anti-FLAG antibodies, and correlated with the dose of the constructs (Fig. 1). This analysis indicated that T-bet and Ag85B were expressed in the transfected cells compared to the internal $\beta$-actin control. Furthermore, the protein levels coding for T-bet and Ag85B were both elevated with increasing doses of FLAG-T-bet and FLAG-Ag85B (Fig. 1). Therefore, the protein levels were closely correlated with the dose of FLAG-T-bet and FLAG-Ag85B.

T-bet enhances Ag85B-specific antibody response and stimulates the secretion of cytokines. To analyze the immunogenicity of T-bet-Ag85B and Ag85B in mice, the groups of BALB/c mice were co-immunized with $100 \mu \mathrm{g}$ pcDNA3.1-FLAG-Tbet in combination with $100 \mu \mathrm{g}$ pcDNA3.1-FLAG-Ag85B, and the immune responses were compared to those induced by immunization with $100 \mu \mathrm{g}$ of pcDNA3.1-FLAG-Ag85B alone. Two weeks after the last immunization, serum antibody titers were detected by ELISA. IgG1 and IgG2a indicates a Th2- and Th1-type immune response, respectively. No specific anti-Ag85B antibodies were detected in the empty vector- and T-bet-immunized mice (Fig. 2). The anti-Ag85B antibody titers in the mice immunized with T-bet-Ag85B and Ag85B alone were markedly enhanced. In addition, IgG1 titers in the mice immunized with Ag85B alone were significantly higher than IgG2a in the Ag85B-immunized mice and also higher than IgG1 in the T-bet-Ag85B-immunized mice. Conversely, $\mathrm{IgG} 2 \mathrm{a}$ in the mice immunized with T-bet-Ag85B were significantly higher than those in the mice immunized with $\mathrm{Ag} 85 \mathrm{~B}$ alone and higher than IgG1 in the mice immunized with T-betAg85B.

Additionally, we characterized the functional phenotype of antigen-specific T cell responses in mice. Splencytes from immunized mice were tested for the secretion of the cytokines IFN- $\gamma /$ IL-2 (Th1-like) and IL-4/IL-10 (Th2-like) upon re-stimulation with FLAG-Ag85B. Splenocytes from mice immunized

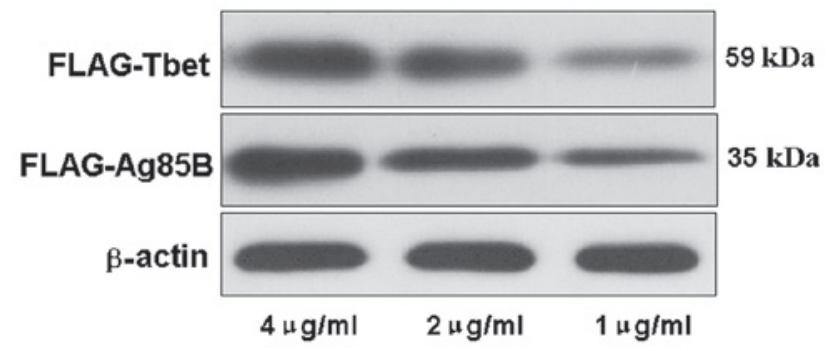

Figure 1. Raw 264.7 cells were transiently transfected with expression vectors coding for FLAG-T-bet and FLAG-Ag85B. The cell lysates were then subjected to SDS-PAGE and western blot analysis using a specific anti-FLAG antibody. There was a dose-dependent expression of FLAG-T-bet and FLAG-Ag85B.

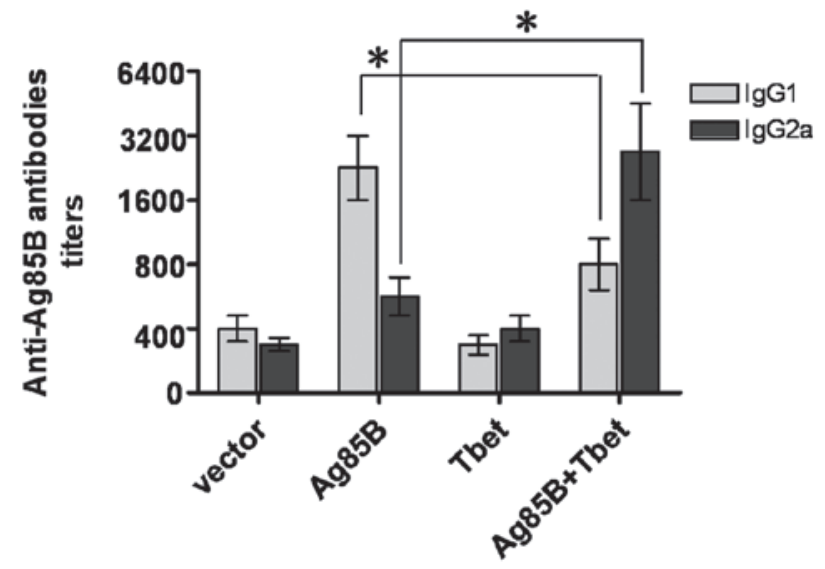

Figure 2. Balb/c mice were vaccinated with emulsified pcDNA3.1-Ag85B, pcDNA3.1-T-bet-Ag85B and a pcDNA3.1 empty vector. Two weeks after the last immunization, the mice were sacrificed and their sera were prepared. The titers of serum IgG1 and IgG2a against MTB in immunized mice were characterized by ELISA. IgG2a titers of mice immunized with Ag85B-T-bet were significantly higher than those of mice immunized with Ag85B alone. However, Ag85B-T-bet-immunized mice had lower levels of IgG1 compared with Ag85B-immunized mice. Data are expressed as the means \pm SD of each group of mice $\left({ }^{*} \mathrm{P}<0.05\right)$.

with T-bet-Ag85B and Ag85B alone produced higher levels of IFN- $\gamma / \mathrm{IL}-2$ and IL-4/IL-10, respectively (Fig. 3). IFN- $\gamma$ and IL-2 levels produced by splenocytes from T-bet-Ag85Bimmunized mice were significantly higher than those from mice immunized with Ag85B alone. However, IL-4 and IL-10 levels produced by splenocytes from mice immunized with T-bet-Ag85B were significantly lower than those produced by splenocytes of mice immunized with $\mathrm{Ag} 85 \mathrm{~B}$ alone. In T-bet-immunized mice, IFN- $\gamma /$ IL-2 levels were significantly higher than IL-4/IL-10 levels. However, the productions of the above-mentioned cytokines were extremely low and there was no significant difference in the mice immunized with the empty vector. The high levels of IFN- $\gamma$ and IL-2 demonstrate that vaccination with pcDNA3.1-T-bet-Ag85B induces potent Th1 responses in mice.

Protection in the lung and spleen by DNA immunization following MTB H37Rv infection in vivo. Finally, to determine the protection of vaccination with plasmids in the lung and spleen against MTB H37Rv infection, we examined the 

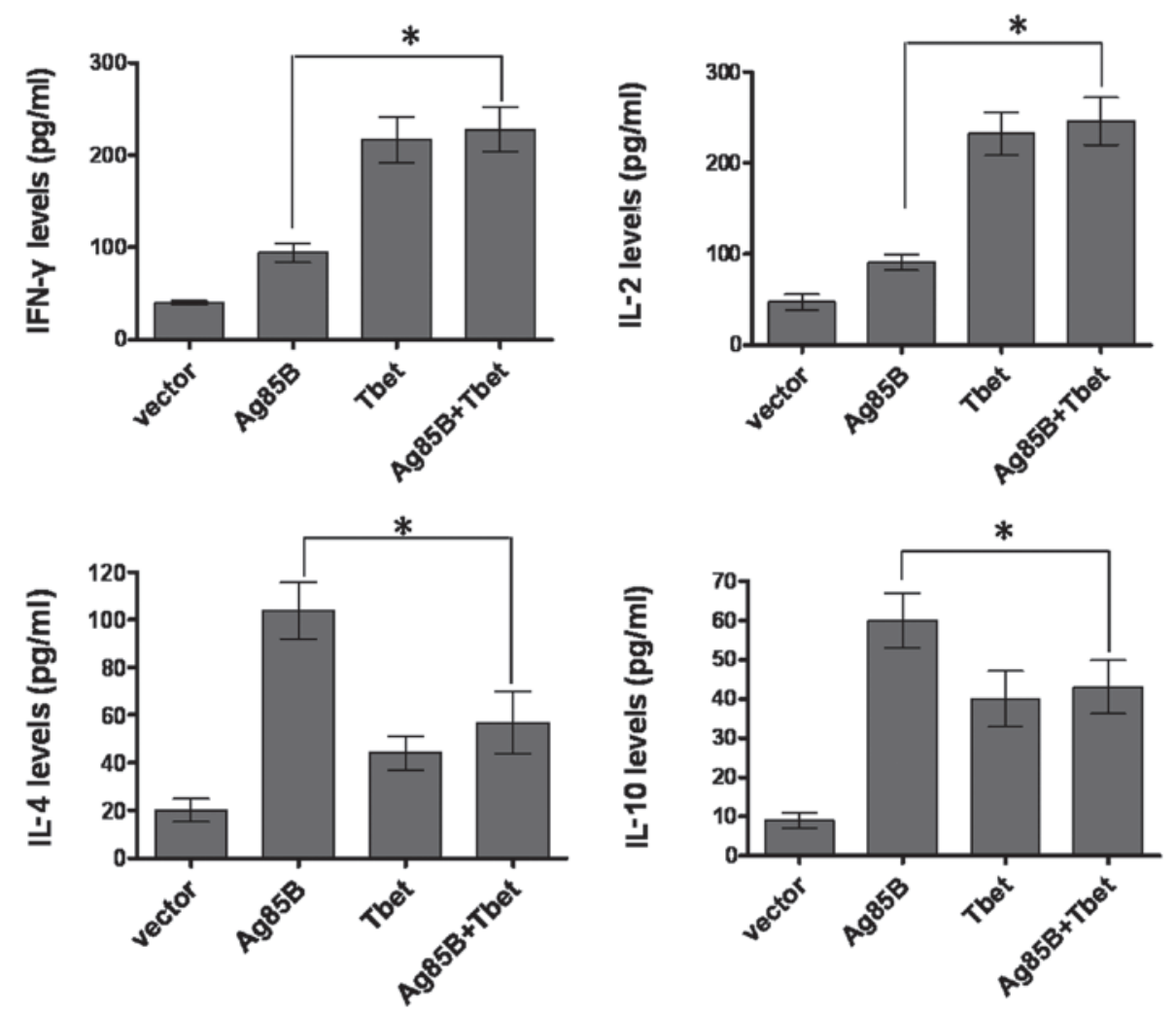

Figure 3. Two weeks after the final immunization, the mice were sacrificed and splenocytes from the vaccinated mice were stimulated with Ag85B for $72 \mathrm{~h}$ and the supernatants were harvested. Then, the concentrations of IFN- $\gamma$, IL-2, IL-4 and IL-10 in the supernatants were determined by the cytokine ELISA assay. The secretion of IFN- $\gamma$ and IL-2 in the pcDNA3.1-T-bet-Ag85B group was significantly higher than in the pcDNA3.1-Ag85B group, while the secretion of IL-4 and IL-10 was significantly lower in the pcDNA3.1-T-bet-Ag85B or pcDNA3.1-T-bet groups than in the pcDNA3.1-Ag85B group. Data are presented as the means $\pm \mathrm{SD}$. However, there was no statistical difference between the pcDNA3.1-T-bet and the vector groups ("P $<0.05)$.
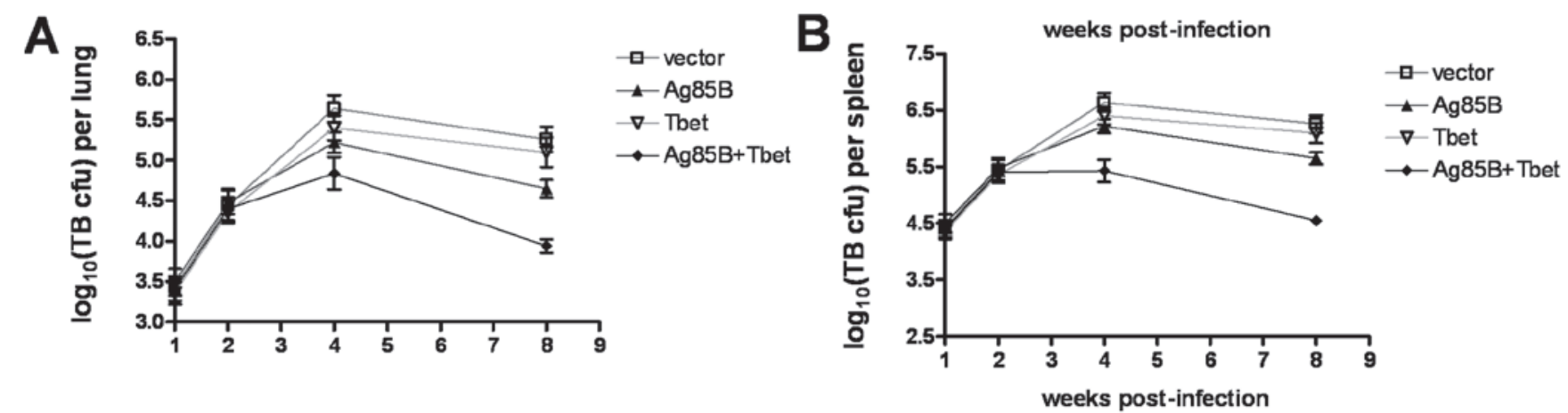

Figure 4. Protection in the lung and spleen by DNA immunization following Mycobacterium tuberculosis (MTB) H37Rv infection. Mice were immunized with the control vector $(\square)$, DNA-Ag85B ( $\mathbf{\Delta})$, T-bet $(\nabla)$ or T-bet + Ag85B $(\bullet), 3$ times, 3 weeks apart. Three months later, the immunized mice were challenged intravenously with 1x10 ${ }^{5}$ CFU MTB H37Rv bacilli. At various times ( $\left.n=5\right)$, the bacterial loads (CFU \pm SEM) were determined in the lung or spleen. At weeks 1 and 2 , in both the lungs and spleens, the bacterial loads were not significantly different among the mice with 4 different treatments. (A) At week 4, DNA Ag85B + T-bet-treated mice had lower loads compared with the vector-treated mice $(\mathrm{P}<0.05)$, while no evident difference was found between DNA Ag85B and Ag85B + T-bet-treated mice in the lungs. At week 8, DNA g85B + T-bet-treated mice had lower loads compared with vector-treated mice $(\mathrm{P}<0.01)$, or compared with DNA Ag85B $(\mathrm{P}<0.05)$ mice in lungs. (B) At week 4, DNA Ag85B + T-bet-treated mice had lower loads compared with vector-treated mice $(\mathrm{P}<0.01)$, or with DNA Ag85B-immunized mice $(\mathrm{P}<0.05)$. At week 8, DNA g85B + T-bet-treated mice had lower loads compared with vector-treated mice $(\mathrm{P}<0.01)$, or compared with DNA Ag85B-treated mice $(\mathrm{P}<0.01)$ in the lungs. The differences of the bacterial growth between the 2 groups of immunized mice were analyzed by the Mann-Whitney U test.

effects of plasmid vaccination on the replication of infected MTB in vivo. Groups of Balb/c mice were vaccinated with pcDNA3.1-T-bet in combination with pcDNA3.1-Ag85B, pcDNA3.1-T-bet, pcDNA3.1-Ag85B and an empty vector. All mice were then challenged with MTB H37Rv and the number of MTB loads in the spleens and lungs were determined by in vitro colony formation assays. At weeks 1 and 2, in the lungs and spleens, the bacterial loads were not significantly different among the mice with the 4 treatments. However, at week 4, the DNA Ag85B + T-bet-treated mice had lower loads compared with the vector-treated mice $(\mathrm{P}<0.05)$, while no evident difference was found between the DNA Ag85B 
and $\mathrm{Ag} 85 \mathrm{~B}+\mathrm{T}$-bet-treated mice in the lungs. In addition, at week 8, DNA Ag85B + T-bet-treated mice had lower loads compared with vector-treated mice $(\mathrm{P}<0.01)$, or compared with the DNA Ag85B-treated mice $(\mathrm{P}<0.05)$ in the lungs (Fig. 4A). In the spleens of the different groups of mice a similar pattern in the number of MTB colonies was detected (Fig. 4B). At week 4, the DNA Ag85B + T-bet-treated mice had lower loads compared with the vector-treated mice $(\mathrm{P}<0.01)$, or with the DNA Ag85B-immunized mice $(\mathrm{P}<0.05)$. Additionally, at week 8, the DNA Ag85B + T-bet-treated mice had lower loads compared with the vector-treated mice $(\mathrm{P}<0.01)$, or compared with the DNA Ag85B-treated mice $(\mathrm{P}<0.01)$ in the spleens. Thus, the Th1 immunity induced by vaccination with recombinant pcDNA-T-bet-Ag85B evidently inhibits the replication of MTB and plays a protective role in the lungs and spleens against MTB infection.

\section{Discussion}

To date, tremendous progress has been made towards the development of novel DNA vaccines against TB (19). In comparison with conventional vaccines, DNA vaccines induce substantial cellular immunity which mainly depends on antigen-specific Th1 responses and is associated with IFN- $\gamma$ and TNF- $\alpha$ production (20). However, studies on the efficiency of DNA vaccines expressing a single MTB antigen in the protection against TB are limited (21). Therefore, appropriate adjuvant selection is essential in improving the protective efficacy of DNA vaccines against TB. Furthermore, T-bet as a specific regulator of immune responses is a good candidate as a DNA adjuvant. Thus, in this study, we selected T-bet as a genetic adjuvant and Ag85B as a model antigen to successfully construct a new composite plasmid, and found that the plasmid, pcDNA3.1-T-bet-Ag85B, induced powerful Ag85Bspecific Th1 responses and repressed the replication of MTB infection in mice.

Firstly, we analyzed MTB Ag85B antigen-specific antibodies in immunized mice and found that vaccination with pcDNA3.1-T-bet in combination with pcDNA3.1-Ag85B elicited higher levels of IgG2a, accompanied by lower levels of IgG1 compared to the pcDNA3.1-Ag85B vaccinated mice. The dominant IgG2a production in Ag85B/T-bet vaccinated mice, demonstrated that vaccination with the $\mathrm{Ag} 85 \mathrm{~B} / \mathrm{T}-\mathrm{bet}$ induced vigorous Th1 responses in mice, and even skewed the Th2-biased immune response established by a protein boost back to a Th1-type response.

Given that MTB-specific Th1 responses and IFN- $\gamma$ and IL-2 production are important in the protection against MTB infection $(1,2)$, in order to confirm whether pcDNA3.1-T-betAg85B can elicit cell-mediated immune responses in vivo, we further detected the levels of cytokines (IFN- $\gamma$, IL-2, IL-4 and IL-10) in the supernatants of the cultured splenic T cells. Our results demonstrated that compared with pcDNA3.1-Ag85B, pcDNA3.1-T-bet-Ag85B and pcDNA3.1-T-bet enhanced the secretion of cytokines IFN- $\gamma$ and IL-2, while the levels of IL-4 and IL-10 decreased. Specificity for Ag85B was confirmed by the fact that the $\mathrm{T}$ cell immune response could not be elicited in the empty vector-immunized mice. Therefore, the Ag85B/T-bet DNA vaccine preferentially induces a Th1-dominant immune response. This further proves that $\mathrm{Ag} 85 \mathrm{~B}$ via T-bet transduc- tion can also be efficiently captured, processed and presented to $\mathrm{T}$ cells, and then mediate a stronger cellular immunity.

Evidently, the therapeutic effectiveness of these new TB vaccines remains controversial in post-exposure models, and certain DNA vaccines can even exacerbate MTB infectionrelated diseases $(22,23)$. The different results may derive from various antigens used for the generation of vaccines. Thus, in this study, we evaluated the protective responses of DNA vaccines in the spleens and lungs following MTB infection. Vaccinated mice were infected with $1 \times 10^{5} \mathrm{CFU}$ of the MTB H37Rv strain. Approximately 3 months later the bacillary burden in the spleens and lungs was detected, and it was identified that the bacterial burdens in Ag85B/T-bet-vaccinated mice were lower than in the empty vector mice. No evident difference between the Ag85B and Ag85B-T-bet mice was observed. Therefore, following MTB infection, treatment Ag85B-T-bet or Ag85B still significantly inhibited the replication of MTB in the lungs and spleens of mice. However, we failed to analyze the MTB infection-related lung inflammation in mice; thus, the therapeutic effectiveness of Ag85B-T-bet vaccines remains uncertain. However, out results did confirm that the Ag85B-T-bet plasmid can be used for the prevention of MTB infection.

Taken together, our data indicate that co-injection of the transcription factor, T-bet, with the Ag85B antigen increases the polarized Th1 immune response, which rebalances the immunity to a Th1 profile, and inhibits MTB replication. Further studies are required for the identification of genes and molecules specifically stimulated or repressed by co-immunization with T-bet and Ag85B that could greatly broaden our understanding of immune correlates of protection in the context of MTB.

\section{Acknowledgements}

This study was supported by the Colleges and Universities Education Grant of Anhui Province (No. 2008jp1042), the Anhui Provincial Natural Science Foundation (No. 1208085QH162) and the National Natural Science Foundation Grants of China (No. 81041083 and No. 81172778).

\section{References}

1. Józefowski S, Sobota A and Kwiatkowska K: How Mycobacterium tuberculosis subverts host immune responses. Bioessays 30: 943-954, 2008.

2. Pai M, Zwerling A and Menzies D: Systematic review: T-cell-based assays for the diagnosis of latent tuberculosis infection: an update. Ann Intern Med 149: 177-184, 2008.

3. Orme IM, Andersen P and Boom WH: T cell response to Mycobacterium tuberculosis. J Infect Dis 167: 1481-1497, 1993.

4. Orme IM: Current progress in tuberculosis vaccine development. Vaccine 23: 2105-2108, 2005.

5. Palma C, Iona E, Giannoni F, Pardini M, Brunori L, Orefici G, et al: The Ag85B protein of Mycobacterium tuberculosis may turn a protective immune response induced by Ag85B-DNA vaccine into a potent but non-protective Th1 immune response in mice. Cell Microbiol 9: 1455-1465, 2007.

6. Doherty TM, Olsen AW, Weischenfeldt J, Huygen K, D'Souza S, Kondratieva TK, et al: Comparative analysis of different vaccine constructs expressing defined antigens from Mycobacterium tuberculosis. J Infect Dis 190: 2146-2153, 2004.

7. Kamath AT, Feng CG, Macdonald M, Briscoe $\mathrm{H}$ and Britton WJ: Differential protective efficacy of DNA vaccines expressing secreted proteins of Mycobacterium tuberculosis. Infect Immun 67: 1702-1707, 1999. 
8. Olsen AW, Williams A, Okkels LM, Hatch G and Andersen P: Protective effect of a tuberculosis subunit vaccine based on a fusion of antigen $85 \mathrm{~B}$ and ESAT-6 in the aerosol guinea pig model. Infect Immun 72: 6148-6150, 2004.

9. Palendira U, Spratt JM, Britton WJ and Triccas JA: Expanding the antigenic repertoire of BCG improves protective efficacy against aerosol Mycobacterium tuberculosis infection. Vaccine 23: $1680-1685,2005$.

10. Glimcher LH: Trawling for treasure: tales of T-bet. Nat Immunol 8: 448-450, 2007.

11. Alcaide P, Jones TG, Lord GM, Glimcher LH, Hallgren J, Arinobu Y, et al: Dendritic cell expression of the transcription factor T-bet regulates mast cell progenitor homing to mucosal tissue. J Exp Med 204: 431-439, 2007.

12. Peng SL: The T-box transcription factor T-bet in immunity and autoimmunity. Cell Mol Immunol 3: 87-95, 2006.

13. Martins GA, Hutchins AS and Reiner SL: Transcriptional activators of helper $\mathrm{T}$ cell fate are required for establishment but not maintenance of signature cytokine expression. J Immunol 175: 5981-5985, 2005.

14. Hu D, Wu J, Hu F, Yang Y, Liang C, Chen J, et al: Stage and tissue specific differences in SjBMI1, a Polycomb protein in Schistosoma japonicum. Parasitol Res 106: 677-682, 2010.

15. Hu D, Wu J, Tang X, Hu F, Yang Y, Du J, et al: Molecular cloning and tissue distribution of a Schistosoma japonicum gene encoding AMY-1. Mol Med Report 4: 1267-1271, 2011.

16. Wu J, Hu D, Yang G, Zhou J, Yang C, Gao Y and Zhu Z: Down-regulation of BMI-1 cooperates with artemisinin on growth inhibition of nasopharyngeal carcinoma cells. J Cell Biochem 112: 1938-1948, 2011.
17. Chen J,Hu X, He S, Wang L, Hu D, Wang X, et al: Expression and immune response analysis of Schistosoma japonicum VAL-1, a homologue of vespid venom allergens. Parasitol Res 106: 1413-1418, 2010.

18. Wu J, Hu D, Du JW, Tao XR, Qi XL and Zhang RB: Th1 immunity is not required for the effect of lipopolysaccharide exposure on modifying asthmatic responses of mice before sensitization. Chin Med J (Engl) 123: 1047-1051, 2010.

19. Hoft DF: Tuberculosis vaccine development: goals, immunological design, and evaluation. Lancet 372: 164-175, 2008.

20. Quesniaux VF, Jacobs M, Allie N, Grivennikov S, Nedospasov SA, Garcia I, et al: TNF in host resistance to tuberculosis infection. Curr Dir Autoimmun 11: 157-179, 2010.

21. Fan X, Gao Q and Fu R: Differential immunogenicity and protective efficacy of DNA vaccines expressing proteins of Mycobacterium tuberculosis in a mouse model. Microbiol Res 164: 374-382, 2009.

22. Taylor JL, Turner OC, Basaraba RJ, Belisle JT, Huygen K and Orme IM: Pulmonary necrosis resulting from DNA vaccination against tuberculosis. Infect Immun 71: 2192-2198, 2003.

23. Turner J, Rhoades ER, Keen M, Belisle JT, Frank AA and Orme IM: Effective preexposure tuberculosis vaccines fail to protect when they are given in an immunotherapeutic mode. Infect Immun 68: 1706-1709, 2000. 